\title{
The Effect of Adenotonsillectomy on Quality of Life in Adults and Pediatric Patients
}

\author{
Soheila Nikakhlagh • Fakher Rahim • \\ Hatam Boostani · Seyed Taghi Beheshti Shirazi • \\ Nader Saki
}

Received: 23 January 2010/Accepted: 13 June 2010/Published online: 11 April 2011

(C) Association of Otolaryngologists of India 2011

\begin{abstract}
The aim of this study was to evaluate the benefits, impact, and overall efficacy of tonsillectomy or adenotonsillectomy on quality of life in patients with recurrent, chronic tonsillitis or adenotonsillar hypertrophy before and after surgery in adults and children. A multicenter, observational, retrospective study of all adults and children who had undergone tonsillectomy in adults (14 years or older) and adenotonsillectomy in children for chronic, recurrent tonsillitis or adenotonsillar hypertrophy between September 2003-September 2008 in Ahwaz Imam and Apadana Hospitals. Patients were asked by questionnaire to compare their symptoms 6-months period before and after tonsillectomy or adenotonsillectomy. Outcome measures included the frequency of tonsillitis per year, days off work (or school), doctor visits and feelings of well-being. 812 adults and children were sent a questionnaire regarding their symptoms in the 6 months before and 6 months after surgery. A total of 812 patients (81 adults and 731 children) were enrolled in the study. The age of the patients ranged from 3 to 42 years. In adults group there were 34 men and 47 women. In child group there were 325 boys and 406 girls. The mean age of the children patients was 8.6 years and adults 26.5 years. The mean duration of
\end{abstract}

S. Nikakhlagh · S. T. B. Shirazi · N. Saki $(\square)$

Department of ENT, Imam Hospital, Ahvaz Jundishapur

University of Medical Sciences, Ahvaz, Iran

e-mail: acrc1387@gmail.com

F. Rahim

Endocrine and Metabolism Research Institute, Tehran University of Medical Sciences, Tehran, Iran

H. Boostani

Psychology Department, Golestan Hospital, Ahvaz Jundishapur

University of Medical Sciences, Ahvaz, Iran symptoms was 3.6 years. The mean frequency of tonsillitis per year, days off work (or school), doctor visits decreased postoperatively. We concluded that tonsillectomy in adult and adenotonsillectomy in children is benefit for patients with adenotonsillar hypertrophy and/or recurrent, chronic tonsillitis and results in significant improvement in overall quality of life, physical health and general well-being.

Keywords Quality of life - Tonsillectomy · Chronic tonsillitis · Adenotonsillar hypertrophy $\cdot$ Patient satisfaction · Questionnaires

\section{Introduction}

The hypertrophy, recurrent and chronic infections of the palatine tonsils and adenoids, is one of the most common problems in the otolaryngology clinic [1]. It is responsible for several alterations on children's development and behavior, what worries parents, and tonsillectomy or adenotonsillectomy (T\&A) remains a commonly performed surgical procedure for both children and adults. In the mid-20th century, over a million tonsillectomies a year were performed on children and adult in the many countries [2,3]. Tonsillectomy for recurrent tonsillitis is effective at significantly reducing the number and severity of sore throats in children and adults who are severely affected. There is also anecdotal evidence that some children's quality of life is transformed by the surgery. This may be caused by a combination of factors that include the tendency of the frequency of recurrent sore throats to resolve over time and the elimination of a source of infection and of obstructive symptoms $[4,5]$. Chronic tonsillitis and recurrent tonsillitis are the most common indication for adult tonsillectomy. Chronic tonsillitis is poorly defined but may be the appropriate term for sore throat of at least 3 months' 
Table 1 Assessment of clinical improvement and quality of life before and after tonsillectomy or adenotonsillectomy

\begin{tabular}{lccc}
\hline Disease severity variables & 6 months before tonsillectomy mean $( \pm \mathrm{SD})$ & 6 months after tonsillectomy mean $( \pm$ SD) & $P$ values \\
\hline Frequency of tonsillitis per year & $8.33(3.45)$ & $1.41(1.75)$ & $<0.005$ \\
Days off work (or school) & $8.78(6.75)$ & $0.61(1.39)$ & $<0.005$ \\
Doctor visits & $5.6(5.7)$ & $8.4(0.6)$ & $<0.001$ \\
Feelings of well-being & $0.5(1.1)$ & $(11.12)$ & $<0.001$ \\
\hline
\end{tabular}

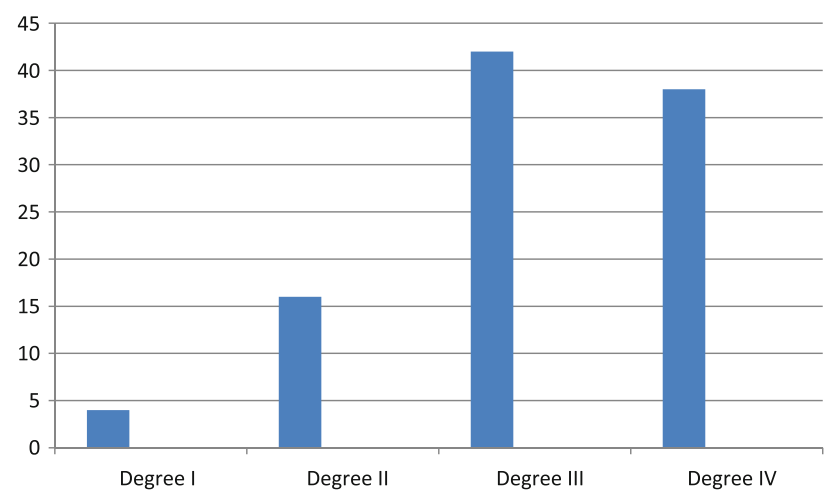

Fig. 1 Pharyngeal tonsil-Percentage of distribution of pharyngeal tonsils according to its size grading

duration accompanied by tonsillar inflammation. Tonsillectomy alone is performed in adult and adenotonsillectomy frequently in children older than 3 years old, whereas adenoidectomy alone is performed infrequently in individuals older than 14 [6]. The rate of adenoidectomy is about 1.5 times as high in boys as in girls, while the rate of tonsillectomy is almost twice as high in girls as in boys [1,7,8]. The aim of this study is to make a comparison between pre- and postoperative signs and symptoms, by showing the results from tonsillectomy or adenotonsillectomy on the quality of life of patients (Table 1, Fig. 1).

\section{Material and Method}

A multi-center, observational, retrospective study of all adults (14 years or older) and children who had undergone tonsillectomy in adults and adenotonsillectomy in children for chronic, recurrent tonsillitis or adenotonsillar hypertrophy between September 2003-September 2008 in Ahwaz Imam and Apadana Hospitals. Parents were applied a questionnaire. The questionnaire referred to the symptoms and effects related to the disease previously to surgery (in the last medical visit before surgery) and the 6-month postoperative followup. The questionnaire was not necessarily applied by the same team which performed surgery. Patients were asked by same questionnaire to compare their symptoms 6-months period before and after tonsillectomy or adenotonsillectomy. Outcome measures included the frequency of tonsillitis per year, days off work (or school), doctor visits and feelings of well-being. 812 adults and children were sent a questionnaire regarding their symptoms in the 6 months before and 6 months after surgery. Inclusion criteria were based on consensus of expert opinion and as follows: recurrent tonsillitis (three or more documented episodes of tonsillitis) in the 6 months prior to enrollment, or chronic tonsillitis as defined by 3 months of sore throat with or without halitosis not responding to appropriate antibiotic therapy. Exclusion criteria were as follow: immunodeficiency, craniofacial anomaly, suspected tonsil malignancy, coagulopathies.

\section{Results}

A total of 812 patients ( 81 adults and 731 children) were enrolled in the study. The age of the patients ranged from 3 to 42 years. In adults group there were 34 men and 47 women. In child group there were 325 boys and 406 girls. The mean age of the children patients was 8.6 years and adults 26.5 years. The mean duration of symptoms was 3.6 years. A review of 812 patients revealed that, following tonsillectomy and/or adenotonsillectomy amount of time taken off work or school due to sore throat reduced from a mean of $8.78( \pm 6.75)$ to $0.61( \pm 1.39)$ days, frequency of tonsillitis per year decreased from $8.33( \pm 3.45)$ to 1.41 $( \pm 1.45)$ days off work (or school), doctor visits decreased from $5.6( \pm 5.7)$ to $0.4( \pm 0.6)$ postoperatively, and more than 91 percent reported improved feelings of well-being, general health and energy. $92.1 \%$ would recommend tonsillectomy to family or friends if they had recurrent tonsillitis. $6.1 \%$ of patients noticed postoperative voice changes. There was a significant improvement on the quality of life of the adenotonsillectomized or tonsillectomized patients, which was confirmed through statistical validation $(P<0.05)$ in $t$-student test. The authors of this commentary have no conflicts of interests to declare.

\section{Discussion}

Our study adds to other studies that showed tonsillectomy is effective in adult patients with recurrent tonsillitis. We 
found statistically significant changes in both disease-specific and quality of life (QOL) after tonsillectomy in adult patients. A review of 100 patients revealed that, following tonsillectomy: their days off work due to sore throat reduced by $95.3 \%$ (from a mean of 27.6 to 1.3 days; confidence intervals 89.7 and $99.6 \%$ ); their visits to their general practitioner due to sore throat reduced by $95.6 \%$ (from a mean of 7.9 to 0.4 visits; confidence intervals 93.8 and 95.1 per cent); and more than $90 \%$ reported improved feelings of well-being, general health and energy.

On average, patients had 8.1 different sore throat episodes, 42 sore throat days, 21.4 days of sore throat related absence from work or school, and 5.9 visits to the GP in the 12 months before their operation. For the 12 months after surgery, these reduced to 0.9 episodes, 4 days, 2.2 days and 0.6 visits, respectively. This reduction was very significant $(P<0.001)$. After their surgery, more than half the patients achieved complete resolution of all the measured parameters mentioned above. Most of the remaining patients achieved at least 50 percent resolution. Only three patients $(4.8 \%)$ achieved less than 50 per cent resolution. Ninety-five percent of the patients found the operation effective in curing their sore throats and were glad they had had surgery. In conclusion, retrospective questionnaire data must be interpreted with some caution, but this study suggests that tonsillectomy is effective in reducing the incidence, duration and co-morbidity of recurrent sore throats in adults; this must be balanced against the postoperative problems in a minority of patients [1-3].

The patients exhibited a mean improvement in quality of life of $27.54 \pm 4.63$ after tonsillectomy according to the Glasgow Benefit Inventory. In the 12 months following the procedure, tonsillectomy resulted in yearly mean decreases in number of weeks on antibiotics by 5.9 weeks, number of workdays missed because of tonsillitis by 8.7 days, and physician visits for tonsillitis by 5.3 visits. In considering the medical costs of tonsillectomy only, the break-even point was found to be 12.7 years, whereas considering the overall economic impact of tonsillectomy resulted in a break-even point of 2.3 years after the procedure.

Flanary et al. evaluated the quality of life in patients with adenotonsillar hypertrophy and obstractive sleep apnea using general quality of life instruments as well as disease-specific instruments. The study showed that quality of life in children with OSA does improve after adenotonsillectomy [9].

Financial Support Research Deputy of Ahwaz Jondishapour University of Medical Sciences.

Conflict of interest None.

\section{References}

1. Ersoy B, Yücetürk A, Taneli F, Ürk V, Uyanik B (2005) Changes in growth pattern, body composition and biochemical markers of growth after adenotonsillectomy in prepubertal children. Int $\mathrm{J}$ Pediatr Otorhinolaryngol 69:1175-1181. doi:10.1016/j.ijporl.2005. 02.020

2. Derkay C, Darrow D, Welch C, Sinacori J (2006) post-tonsillectomy morbidity and quality of life in pediatric patients with obstructive tonsils and adenoid: microdebrider vs eletrocautery. Otolaryngol Head Neck Surg 134:114-120. doi:10.1016/j. otohns.2005.10.039

3. Mitchell R, Kelly J (2006) Long-term changes in behavior after adenotonsillectomy for obstructive sleep apnea syndrome in children. Otolaryngol Head Neck Surg 134:374-378. doi:10.1016/ j.otohns.2005.11.035

4. Mitchell R, Kelly J (2005) Quality of life after adenotonsillectomy for SDB in children. Otolaryngol Head Neck Surg 133:569-572. doi:10.1016/j.otohns.2005.05.040

5. Glover JA (2008) The incidence of tonsillectomy in school children. Int J Epidemiol 37(1):9-19. doi:10.1093/ije/dym258

6. Alho OP, Koivunen P, Penna T, Tepp H, Koskela M, Luotonene J (2007) Tonsillectomy versus watchful waiting in recurrent streptococcal pharyngitis in adults: randomized controlled trial. BMJ 334:939

7. Burton MJ, Glasziou PP (2009) Tonsillectomy or adeno-tonsillectomy versus non-surgical treatment for chronic/recurrent acute tonsillitis (Review). evidence-based child health. Cochrane Rev J 4:1291-1326

8. Russo CA, Owens P, Steiner C, Josephsen J. Ambulatory Surgery in U.S. Hospitals, 2003-HCUP Fact Book No. 9. AHRQ Publication No. 07-0007, January 2007. Agency for Healthcare Research and Quality, Rockville, MD. http://www.ahrq.gov/ data/hcup/factbk9/

9. Flanary VA (2003) Long-term effect of adenotonsillectomy on quality of life in pediatric patients. Laryngoscope 113(10): 1639-1644 [PMID: 14520088] 